\title{
Two Models of Magnetic Support for Photoevaporated Molecular Clouds
}

D.D. Ryutov, J.O. Kane, A. Mizuta, M.W. Pound, B.A. Remington

May 6, 2004

Astrophysics and Space Science 
This document was prepared as an account of work sponsored by an agency of the United States Government. Neither the United States Government nor the University of California nor any of their employees, makes any warranty, express or implied, or assumes any legal liability or responsibility for the accuracy, completeness, or usefulness of any information, apparatus, product, or process disclosed, or represents that its use would not infringe privately owned rights. Reference herein to any specific commercial product, process, or service by trade name, trademark, manufacturer, or otherwise, does not necessarily constitute or imply its endorsement, recommendation, or favoring by the United States Government or the University of California. The views and opinions of authors expressed herein do not necessarily state or reflect those of the United States Government or the University of California, and shall not be used for advertising or product endorsement purposes. 


\title{
TWO MODELS OF MAGNETIC SUPPORT FOR PHOTOEVAPORATED MOLECULAR CLOUDS
}

\author{
D.D. Ryutov ${ }^{1}$, J.O. Kane ${ }^{1}$, A. Mizuta ${ }^{2}$, M.W. Pound ${ }^{3}$, B.A. Remington ${ }^{1}$ \\ ${ }^{1}$ Lawrence Livermore National Laboratory, Livermore, CA 94551, USA \\ ${ }^{2}$ Institute of Laser Engineering, Osaka University, Osaka, 565-0871, Japan \\ ${ }^{3}$ Astronomy Department, University of Maryland, College Park, MD 20742, USA
}

\begin{abstract}
The thermal pressure inside molecular clouds is insufficient for maintaining the pressure balance at an ablation front at the cloud surface illuminated by nearby UV stars. Most probably, the required stiffness is provided by the magnetic pressure. After surveying existing models of this type, we concentrate on two of them: the model of a quasi-homogeneous magnetic field and the recently proposed model of a "magnetostatic turbulence". We discuss observational consequences of the two models, in particular, the structure and the strength of the magnetic field inside the cloud and in the ionized outflow. We comment on the possible role of reconnection events and their observational signatures. We mention laboratory experiments where the most significant features of the models can be tested.
\end{abstract}

\section{Introduction}

The complex shapes of photoevaporated molecular clouds (e.g., the Eagle Nebula and the Horsehead Nebula) are thought to be created by the ablation pressure of the ionized outflows (e.g., Spitzer, 1978). [The ablation is caused by the ionizing radiation of the nearby young stars.] The material inside the clouds is very cold, with the temperature of order of 10-30 K. So low a temperature is explained by that, at the temperature exceeding, roughly, $50 \mathrm{~K}$, the radiation in molecular transitions (Neufeld et al, 1995) becomes so intense that the cooling time reaches a very small value $\sim 100$ years, whereas the dynamical time of the typical molecular cloud exceeds $10^{5}$ years. The ablation pressure drives compression waves into the cloud interior. However, the shock and compressional heating cannot compete with the cooling and the cloud interior stays at low temperatures, in the range 10-30 K.

TABLE 1. Parameters of the Eagle Nebula

\begin{tabular}{|c|c|c|c|c|c|c|}
\hline Parameter* & $L, \mathrm{~cm}$ & $\tau, s$ & $\mathrm{v}^{\prime}, \mathrm{s}^{-1}$ & $n_{H 2}, \mathrm{~cm}^{-3}$ & $T_{0}, \mathrm{~K}$ & $x$ \\
\hline $\begin{array}{c}\text { Numerical } \\
\text { value }\end{array}$ & $10^{18}$ & $3 \times 10^{12}$ & $3 \times 10^{-13}$ & $5 \times 10^{4}$ & 30 & $10^{-8}$ \\
\hline
\end{tabular}

*Notation: $L$ - characteristic spatial scale (the diameter of a pillar II, Pound, 1998); $\tau$ - characteristic temporal scale; $\mathrm{v}^{\prime}$ characteristic velocity gradient, $n_{H 2}$ - average density of the molecular hydrogen; $T_{0}$ initial cloud temperature, $x$ - characteristic ionization degree.

We will make all the numerical estimates for the case of the Eagle Nebula, which is relatively well characterized compared to other similar objects [Interesting data pertaining to the Horsehead Nebula can be found in Pound et al, 2003.] The most important parameters are presented in Table 1, compiled on the basis of Spitzer, 1978; Hester et al, 1996; Pound, 1998; Levenson et al, 2000; see also a summary in Ryutov et al, 2004. The ionization degree of the cloud is $\sim 10^{-8}$ and is determined by the ionization by cosmic rays and photoemission from the dust grains (Elmegreen, 1998). [The cloud consists predominantly of the molecular hydrogen, with some admixture of molecules like CO and water, as well as dust particles, made of carbon and silicates, e.g., Spitzer, 1978].

Despite so low an ionization degree, the skin time for the cloud is much longer than the dynamical time (see Sec. III). In other words, the line-tying of the magnetic field is a reasonable concept, at least at the level of the first rough models. 
The amount of ionized gas evaporated from the surface per unit time is determined by the intensity of the ionizing continuum reaching the cloud surface. The resulting outflow has a temperature of order of $10^{4} \mathrm{~K}$ and the density $\sim 10^{3} \mathrm{~cm}^{-3}$ (Hester et al, 1996; Pound, 1998; Levenson et al, 2000).

TABLE 2. Parameters of the ionized outflow

\begin{tabular}{|c|c|c|c|c|}
\hline Parameter** & $\mathrm{v}_{\mathrm{a}}, \mathrm{cm} / \mathrm{s}$ & $T_{\alpha}, \mathrm{K}$ & $n_{a}, \mathrm{~cm}^{-3}$ & $\begin{array}{c}P_{a}, \mathrm{CGS} \\
\left(\mathrm{Kelvins} / \mathrm{cm}^{3}\right)\end{array}$ \\
\hline $\begin{array}{c}\text { Numerical } \\
\text { value }\end{array}$ & $3 \times 10^{6}$ & $10^{3}$ & $10^{3}$ & $1.6 \times 10^{-8}\left(10^{8}\right)$ \\
\hline
\end{tabular}

** $\mathrm{v}_{a}, T_{a}, n_{a}$ are the velocity, the temperature, and the electron density in the ablation outflow; $p_{a}$ is the ablation pressure The quantities related to the ablation outflow bear a subscript " $a$."

From Tables 1 and 2, one sees that the ablation pressure is almost two orders of magnitude higher than the gaseous pressure inside the cloud. This means that the cloud should have collapsed to much higher densities than those actually observed. The emerging problem can be called the problem of "missing stiffness" (Ryutov et al, 2004). Here we discuss two possible models that may explain the paradox: the model of a quasi-uniform magnetic field, and the model of magnetostatic turbulence. The main focus of our paper is the identification of the observational consequences that may help in determining the validity of the models. We also briefly discuss possible laboratory experiments.

$a$

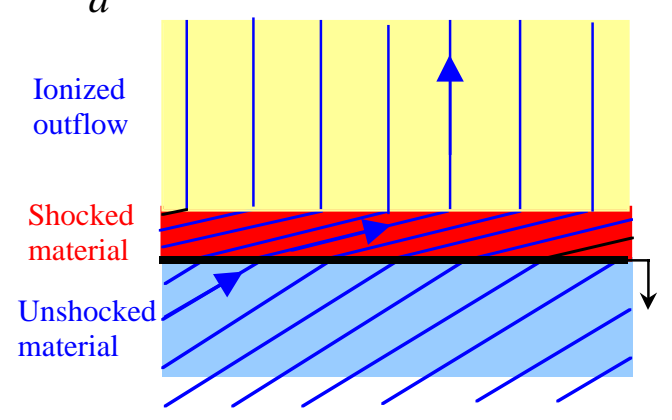

$b$

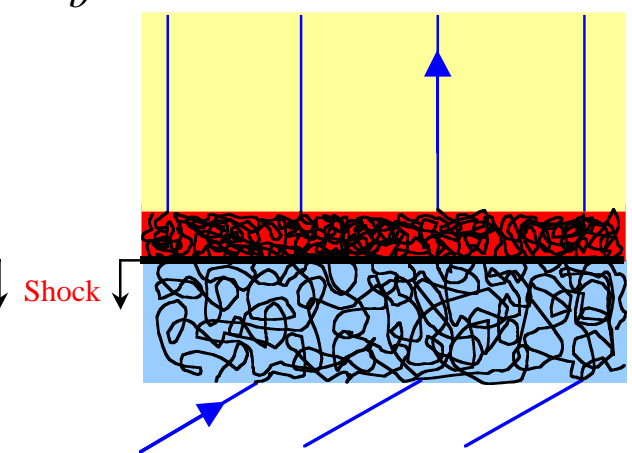

Fig. 1. Two models of magnetic support for a slab model of the cloud illuminated from above: a) A homogeneous magnetic field is initially tilted with respect to the cloud surface; when the ionizing radiation "turns on," an ablation outflow is formed; the tangential component is compressed in the shocked material; the lowest slab represents the unsocked material with initial magnetic field. b) A random small-scale magnetic field. In this model, the net magnetic flux permeating the cloud is very small; the magnetic field in the ionized outflow is weak and does not have any dynamical significance. It may have a more complex structure than shown.

\section{The Model of a Quasi-Homogeneous Magnetic Field}

This model is based on the assumption that there is a large-scale primordial magnetic field permeating the cloud. In the past, this assumption was analyzed mainly in terms of its effect on the star formation (e.g., McKee et al, 1993, and references therein). We will consider the consequences of this assumption in terms of the effect of the magnetic field on the formation of the observed large-scale structures (the pillars). To make some preliminary estimates, we consider a slab model shown in Fig. 1. In order to provide stiffness with respect to the compression by the ablation pressure, the initial magnetic field has to have a substantial component parallel to the surface. On the other hand, there is no reason to believe that the magnetic field would be perfectly aligned with the surface. So, we make a natural assumption that it initially intersects the cloud surface at an angle of order 1, i.e., that 
the normal and tangential components are initially ocomparable, $B_{n 0} \sim B_{t 0}$ (Fig. 1a) When the ablation pressure "turns on," the tangential component of the magnetic field inside the cloud is compressed to some value $B_{t}$ to provide the balance with the ablation pressure; in other words, the condition $B_{t}^{2} / 8 \pi \sim p_{a}$ is reached. Here we neglect the contribution of the gaseous pressure, which is small (see Introduction). Assuming the density compression ratio to be $\sim 3$ (compatible with the observations, Pound, 1998), one finds, using the value of $p_{a}$ from Table 2 , that the initial tangential magnetic field, $B_{t 0} \sim(1 / 3) B_{t} \sim(1 / 3) \sqrt{8 \pi p_{a}}$, should be $\sim 150 \mu \mathrm{G}$. For $B_{n 0} \sim B_{t 0}$, this mean that the total initial magnetic field, $B_{0}=\sqrt{B_{n 0}^{2}+B_{t 0}^{2}}$, should be $\sim 200$ $\mu \mathrm{G}$. Unfortunately, there are no direct measurements of the magnetic field for the Eagle Nebula. Judging from Crutcher's (1999) survey of the magnetic field measurements for analogous objects, this value is somewhat high, but not so high as to rule it out.

Consider now the magnetic field in the ionized outflow, just beyond the ionization front. The normal component of the magnetic field does not change when the gas passes through the ionization front and expands, i.e., $B_{n a} \sim B_{n 0}$ (as before, the subscript $a$ designates the field in the ablation flow). Conversely, the tangential component varies in proportion to the density, $B_{t a} \sim\left(n_{a} / n_{H_{2}}\right) B_{t 0}$. Taking the densities from Tables 1 and 2 , one finds that the tangential component decreases by a factor of 50 compared to its initial value. Therefore, the magnetic field lines in the ionized outflow will be directed almost normally with respect to the surface of the ablation front. This conclusion, obviously, holds also in the case of a nonplanar surface of the column. In other words, if the model of a quasi-homogeneous magnetic field is correct, it predicts that the magnetic field in the ablation outflow near the cloud surface must be essentially normal to the surface. This conclusion is illustrated by Fig. 2 .

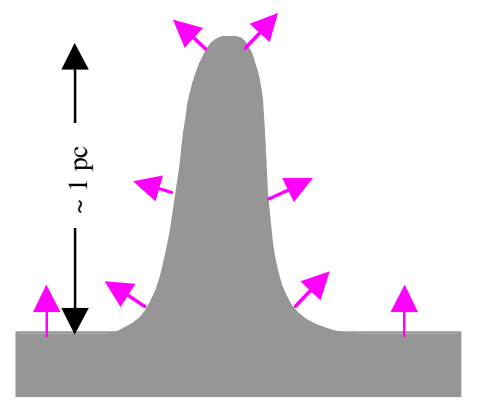

Fig. 2. In the quasi-homogeneous model, the magnetic field just outside the cloud must be directed along the normal to the cloud surface

As we have already mentioned, direct measurements of the magnetic field around the Eagle Nebula are not available. As an indirect indication of a possible presence of the perpendicular magnetic field one may consider the presence of fine non-uniformities in the outflow visible at high-resolution images: these non-uniformities are indeed almost perpendicular to the surface.

The $B_{n a}$ value in the case of the Eagle Nebula must be approximately $150 \mu \mathrm{G}$. The corresponding magnetic pressure is significantly less than the ablation pressure, so that the magnetic field does not have any significant dynamical effect on the outflow. The field lines down the stream are shaped according to the line-tying constraint, i.e., they are stretched along the streamlines.

The difficulty with the model of the quasi-uniform magnetic field is that such a field would favor development of 2D structures of the type of ridges, not the 3D column-like structures present in the Eagle Nebula (Fig. 3). This is due to the anisotropic nature of the Maxwell stress tensor: The field lines of the magnetic field frozen into conducting medium 
would be pulled out together with the pillar material as shown in Fig. $3 b$ and create a strong tension that would force the column to fall back. Conversely, in the case of a ridge-like structure aligned with the direction of the magnetic field (Fig. 3a) such a tension is absent.
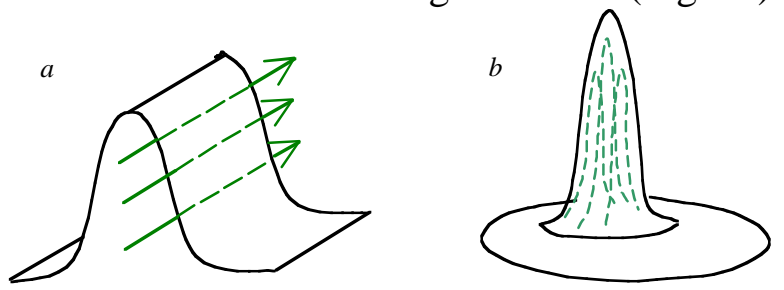

Fig. 3 Formation of a ridge aligned with the magnetic field (panel a) does not cause generation of the returning force, whereas formation of a column (panel b) does. The normal component of the magnetic field is not shown, because it plays a relatively minor dynamical role.

This apparent contradiction can be solved by allowing for the magnetic reconnection. Indeed, if such a process near the base of the column occurs (Fig.4 a,b), the overall returning force acting on the column significantly decreases. Reconnection events may occur several times in the course of the pillar growth, generating a structure shown in Fig.4c

The reconnection time $\tau_{\text {rec }}$ must be at least a few times less than the dynamical time $\tau$. The magnetic flux annihilated in each reconnection event is of order of $L^{2} B_{t 0}$. The loop voltage that develops during the reconnection is $\mathrm{V}_{\text {loop }} \sim L^{2} B_{t 0} / c \tau_{\text {rec }}$. Assuming that $\tau_{\text {rec }} \sim 0.3 \tau$, $B_{t 0} \sim 150 \mu \mathrm{G}$ and taking the other numbers from Table 1, one finds that the loop voltage is very large, $\sim 5 \times 10^{9} \mathrm{CGS} \sim 1.5 \times 10^{12} \mathrm{~V}$. In other words, reconnections may lead to generation of the cosmic rays. Evaluation of the relative significance of this source with respect to the other known sources of cosmic ray would require collecting the statistics of photoevaporated molecular clouds.
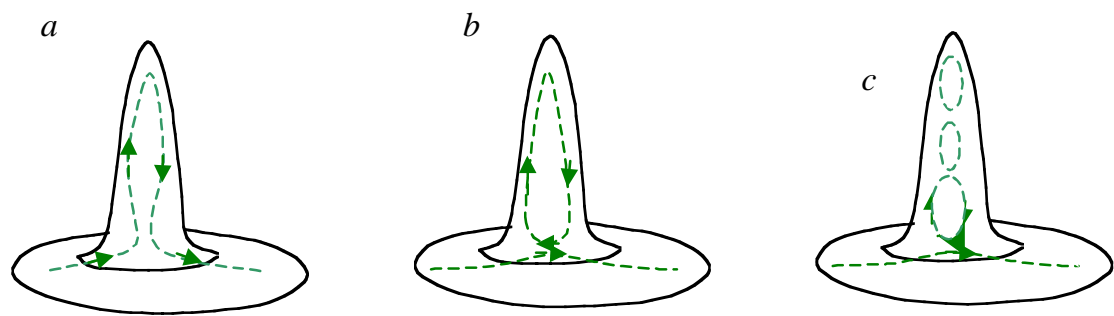

Fig. 4. Reconnections near the base of the column may disconnect the field lines In the lower and the upper parts of the column, thereby reducing the returning force; the force reduction is especially strong if multiple reconnections occur (c).

The electric field $E \sim \mathrm{V}_{\text {loop }} / L$ that develops during the reconnection is large enough to cause a breakdown of the molecular hydrogen. The possibility of gas breakdown in reconnection events was discussed in Birk et al, 2004, in conjunction with low-density dif9use clouds as a mechanism for sustaining the observed ionization degree of such clouds. We note that, because of the higher density of photoevaporated molecular clouds, the breakdown will be accompanied by intense X-ray radiation. The part beyond a few $\mathrm{keV}$ would be only weakly absorbed by the cloud material and could, in principle, be detected by the external observer. As is known, e.g., from observation of reconnections in the Solar atmosphere (Title, 2004), the reconnection often leads to formation of significant current concentrations of the type of filaments and knots. If our model is correct, the X-ray radiation will manifest such features.

\section{The Model of Magnetostatic Turbulence}

We now consider a model in which the magnetic field inside the cloud is random, with the r.m.s. value of this random field being much greater than the value of any possibly 
present quasi-homogeneous component, $\left.\left.\left(\left\langle B^{2}\right\rangle\right)^{1 / 2}\right\rangle\right\rangle|\langle\boldsymbol{B}\rangle|$, Fig. 1b, and the scale-length $l$ of the random field being much less than the global scale $L$. In order to be relevant in the problem of "missing stiffness," the magnetic pressure of the random magnetic field has to be comparable to the ablation pressure and, therefore, much higher than the gaseous pressure (see Sec. 1). This causes a problem: the MHD turbulence driven by so strong a magnetic field is necessarily supersonic and, as shown in McLow et al. (1998), Stone et al. (1998), Ostriker et al. (2001) decays very rapidly, within one turn-over time of the eddies, $l / \mathrm{v}_{\mathrm{A}}$, with $\mathrm{v}_{\mathrm{A}}^{2}=\left\langle B^{2}>/ 4 \pi \rho\right.$. In our problem, the strong radiative cooling of the gas keeps its sound speed at a deeply sub-Alfvenic level, thereby not allowing the turbulence to reach a weakly compressible state (where one might expect a transition to long-lasting turbulence). Therefore, the MHD turbulence would survive for only a very short time $\sim l / \mathrm{v}_{\mathrm{A}}<<\tau$ and wouldn't provide a lasting support for the cloud.

It was pointed out by Ryutov and Remington (2002), that this difficulty can be circumvented if one assumes that the tangled magnetic field is a force-free field, i.e., that the current is everywhere (almost) parallel to the magnetic field, $\boldsymbol{j} \| \boldsymbol{B}$. In such a case, the presence of a tangled magnetic field does not induce strong small-scale motions, in particular, does not generate shocks, and the tangled structure can exist for the times determined by resistive dissipation of the magnetic field (which time is very long). As the presence of a random "turbulent" magnetic field in this case is not associated with rapid turbulent motions of the gas, this state was called in Ryutov and Remington (2002) the state of "magnetostatic turbulence."

Although the magnetostatic turbulence is force-free at small spatial scales, it acts analogously to a gaseous pressure when the compression at a large-scale occurs. For an isotropic turbulence, it acts as a gas with the adiabatic index $\gamma=4 / 3$ (Ryutov and Remington, 2002). The numerical analysis of such a system is presented in Mizuta et al, this issue.

In the magnetic field measurements, an integration over the line of sight is carried out; in addition, the finite spatial resolution causes a smearing over a finite area in the plane of the sky. In the case of small-scale random magnetic field, this causes a significant averaging-out. Therefore, if the model of magnetostatic turbulence works, the measured magnetic field strength should be substantially less than $\sim 200 \mu \mathrm{G}$ required in the model of a quasi-homogeneous field. The weaker field seems to be in a better agreement with Crutcher, 1999.

As the current sustaining magnetostatic turbulence flows predominantly along the magnetic field lines, the resistivity $\eta$ responsible for the dissipation of this state is the parallel resistivity $\eta_{\|}$. For the parameters given in Table 1, this resistivity (we use CGS units) is certainly lower than (in CGS units) $10^{-6} \mathrm{~s}$ (the latter number corresponds to the improbable case where all the electrons are attached to the dust grains and the current in the rest-frame of the fluid is carried solely by the ions; there are all reasons to expect that the resistivity is much lower). Accordingly, the magnetic diffusivity $D_{m}=c^{2} \eta_{\|} / 4 \pi$ is lower than $10^{14}$. Assuming that the scale $l$ of the magnetic structures is $1 / 30$ of $L$, one finds that the resistive dissipation time is many orders of magnitude longer than the dynamic time $\tau$.

The magnetostatic turbulence may be dissipated by the reconnection process. However, the fact that the initial state is almost force-free may slow-down the reconnection rate. Additional "longevity" can appear in the turbulent state in which the parameter $\lambda$ that enters the force-free equation $\nabla \times \boldsymbol{B}=\lambda \boldsymbol{B}$ varies in space slowly, $|\nabla \lambda|<<\lambda^{2}$ : in this case, the magnetic field is locally in the so-called Taylor state (Taylor, 1974), and reconnection is inhibited. The absolute value of $\lambda$ is $\sim l^{-1}$. 
Reconnection events will serve as sources of X-ray radiation, very much like in the case considered in Sec. II. However, these events will now occur in the numerous vortices of the scale $l<<L$. Therefore, one can expect that the external observer will see only diffuse radiation produced by simultaneously occurring small-scale reconnections distributed over the whole volume of the pillars and averaged due to the finite spatial resolution.

\section{Discussion}

Two models of magnetic support that we have discussed in this paper seem both to be compatible with the observed general structure of the Eagle Nebula. However, the predictions regarding the properties of the magnetic field are quite different and may, in principle, be used for discrimination between the models. The models differ also in the predictions related to the 1-10 keV-range x-ray radiation from the clouds: the model of a quasi-homogeneous field predicts the presence of a few bright knots and filaments, whereas the model of magnetostatic turbulence predicts the domination of the diffuse radiation.

Laboratory experiments may help to address key physics issues. In the case of a quasihomogeneous field model, the most interesting issue is that of reconnections occurring during the pillar growth. The corresponding experiment can be performed in the general setting of the experiment described in Yamada et al., 2000. In the case of the model of the magnetostatic turbulence (which predicts that the gas with embedded random force-free magnetic field will behave as a polytropic gas with $\gamma=4 / 3$ ), one can study the hydrodynamics of the ablation front in the setting of the high-energy-density laser experiment (Remington et al, 1993).

Work performed for the U.S. DoE by UC LLNL under contract W-7405-Eng-48 and partly supported by the NASA grant NRA-00-01-ATP-059.

\section{References}

Birk, G.T., Lesch, H., Neukirch, T.: 2004, Astrophys. Space Sci., 289, 359.

Crutcher, R.M: 1999, ApJ, 520, 706.

Elmegreen, B.G.: 1998, In: Astronomical Society of Pacifics Conference Series, “Origins,” Proc. of the Intern. Conf. held at Estes Park, Colorado, May 19-23 1997, v.148, C.E. Woodward, J.M. Shull, H.A. Thronson, Jr. - Eds., p150.

Hester, J.J., Scowen, P.A., Sankrit, R. et al: 1996, Astronomical Journ. 1112349.

Levenson, N., Graham, J.R., McLean, I.S., et al: 2000, ApJ, 533, L53.

McKee, C.F. Zweibel, E.G., Goodman, A.A., \& Heiles, C.: 1993, in Protostars and Planets III, ed. E.H. Levy \& J.L. Lunine (Tucson: Univ. Arizona Press), 327.

Mac Low, M.-M., Klessen, R.S., A. Burkert, A., Smith, M.D.: 1998, Phys. Rev. Lett., 80, 2754.

Mizuta, A et al, this issue

Neufeld, D.A., Lepp, S, Melnick G.J.: 2000, ApJ Supplement 100, 132.

Pound, M.W.: 1998, ApJ, 493, L113.

Pound, M.W., B. Reipurth, J. Bally 2003, Astronomical Journ. 125, 2108.

Remington B A, Weber S V, Haan S W, et al.: 1993 Phys. Fluids $\mathbf{B 5} 2588$.

Ryutov, D.D., Remington, B.A.: 2002, Plasma Phys. Contr. Fus., 44, B407.

Ryutov, D.D., Kane, J.O., Mizuta, A., et al.: 2004. In: "Plasmas in the Laboratory and in the Universe”, AIP Conference Proceedings, Volume 703, p. 415, Melville, NY.

Spitzer, L.: 1978, "Physical Processes in the Interstellar Medium” John Wiley \& Sons, New York

Stone, J.M., Ostriker, E.C., Gammie, C.F.: 1998, ApJ 508, L99-L102.

Ostriker, E.C., Stone, J.M., Gammie, C.F.: 2001, ApJ 546, 980.

Taylor, J.B.: 1974, Phys. Rev. Lett. 33, 1139

Title, A., 2004. In: “Plasmas in the Laboratory and in the Universe”, AIP Conference Proceedings, Volume 703, p. 163, Melville, NY, 2004.

Yamada, M., Ji, H., Carter, T. et al.: 2000 Phys. Plasmas 71781. 